\title{
Características Psicométricas de una Rúbrica para Evaluar Expresión Escrita a Nivel Universitario
}

\author{
Carla Muñoz ${ }^{(1) *}$ y Jorge Valenzuela ${ }^{(2)}$ \\ (1) Pontificia Universidad Católica de Valparaíso, Escuela de Pedagogía. Av. El Bosque 1290, Edificio B, \\ Viña del Mar-Chile (e-mail: carla.munoz@ucv.cl) \\ (2) Universidad de Playa Ancha, Centro de Estudios Avanzados, Traslaviña 450, Viña del Mar-Chile \\ (e-mail: jorge.valenzuela@upla.cl)
}

${ }^{*}$ Autor a quien debe ser dirigida la correspondencia

Recibido May. 22, 2015; Aceptado Jun. 17, 2015; Versión final Jul. 7, 2015, Publicado Dic. 2015

\begin{abstract}
Resumen
El objetivo de este artículo es presentar y analizar las características psicométricas de una rúbrica para evaluar expresión escrita a través de un ensayo académico. Para este efecto, se evaluó la producción de un texto académico tipo ensayo en una muestra de 745 estudiantes, de ambos sexos, todos universitarios de primer año, pertenecientes a 17 carreras universitarias, en el contexto de un curso de expresión escrita. El producto escrito fue corregido por medio de la rúbrica por evaluadores capacitados para este fin. Se analizó la estructura factorial y la confiabilidad del instrumento, así como la confiabilidad inter-correctores. Los resultados del análisis factorial exploratorio, aportan evidencia sobre la estructura factorial. Debido a la alta confiabilidad de los índices, la rúbrica puede ser considerada como un instrumento fiable y de fácil aplicación.
\end{abstract}

Palabras clave: escritura académica; rúbrica; pedagogía universitaria; expresión escrita; evaluación

\section{Psychometric Features of a Rubric to Assess Academic Writing at University Level}

\begin{abstract}
The aim of this paper is to present the psychometric properties of a rubric for assessing academic writing at university level. The study evaluated the production of an academic text in a sample of 745 freshmen from 17 academic programs. Through the rubric, trained correctors evaluated the essay. Reliability and factorial structure of the instrument as well as reliability between the test correctors were obtained. The results show that the exploratory factor analysis provides evidence of the factorial structure. Due to the high reliability indices, the rubric can be considered as a reliable and easy applicable instrument.
\end{abstract}

Keywords: academic writing; rubric; higher education; written expression; assessment 


\section{INTRODUCCIÓN}

Las investigaciones ya clásicas sobre los procesos de escritura demuestran con claridad que lo que se creía un don mediado por la inspiración y el talento personales, se trata más bien de una tarea compleja que moviliza una serie de operaciones y recursos cognitivos (Cf. Alamargot \& Chanquoy, 2001; Torrance \& Galbraith, 2006) y, lo que es más importante aún, que puede ser enseñada directamente. En esta línea, muchas son las universidades anglófonas que han instaurado en sus curricula la enseñanza explícita de la escritura o la han integrado en el contexto de un paradigma conocido como "writing across the curriculum" en donde todas las asignaturas del currículo están conectadas por la escritura (Adoumieh, 2014; Bazerman, 2005; Carlino, 2004, 2005; Melas et al., 2015). Vista desde la perspectiva de las teorías clásicas sobre producción escrita, la escritura es un instrumento del pensamiento que permite a quien la utiliza resolver problemas, alcanzando determinados objetivos retóricos (Bereiter \& Scardamalia, 1987).

En habla hispana, sin embargo, la preocupación sobre los procesos de producción escrita en la edad adulta (y en particular, por la escritura académica) es de reciente data (Carlino, 2013). La mayoría de estos estudios ha tenido un carácter diagnóstico y han puesto en evidencia la necesidad de contar con dispositivos pedagógicos que remedien las falencias encontradas en los estudiantes que ingresan al ciclo superior de educación. Sin embargo, si bien existen en Chile algunos instrumentos validados, estos permanecen como reservados a las instituciones que los utilizan. Es el caso de pruebas como la de comunicación escrita de la Universidad Católica de Chile (Flotts \& Manzi, 2012), la prueba CODICE en la Universidad de Chile (Cabrera, 2014) o la medición de habilidades de comunicación escrita en la Prueba Inicia (Cf. Garcés \& Riquelme, 2014; Rodríguez \& Castillo, 2014). Fuera de estos instrumentos, no existen hasta donde hemos podido investigar- instrumentos debidamente validados que permitan la evaluación permanente de estas habilidades. La necesidad de contar con un instrumento válido y confiable que permita al docente evaluar el nivel de escritura al inicio del ciclo formativo universitario, pero que a la vez logre dar información específica al estudiante sobre su nivel real de competencia escritural; nos ha llevado a concebir esta grilla o rúbrica de evaluación de la escritura. El instrumento (de ahora en adelante, la rúbrica) ha sido elaborado sobre la base de los siguientes principios: la rúbrica de expresión escrita como instrumento de evaluación diagnóstica, el ensayo como modelo de escritura y la actividad de escritura como herramienta epistémica.

Teóricamente, la evaluación de la producción escrita debe considerar, al menos, dos dimensiones fundamentales que podríamos denominar "global" y "local", respectivamente. Por una parte, los aspectos globales del texto, esto es, los referidos a la organización y estructura del texto a nivel global y por otra, los aspectos referidos a elementos locales (Conexión entre frases, ortografía puntual y acentual). Ambas dimensiones contribuyen a la riqueza argumentativa del texto. En efecto, gran parte del trabajo cognitivo de la escritura académica se refiere a la carga de la argumentación (van Eemeren, 2001).

La primera dimensión considera aspectos asociados al contenido del escrito o unidad temática del mismo, es decir la existencia de un eje temático central y ausencia de digresiones, así como la organización del texto en una estructura equilibrada que respete la apertura, el desarrollo y el cierre del tema. Respecto de la segunda dimensión del ensayo, debiera considerar la coherencia local del texto, así como aspectos de cohesión y respecto por la norma formal escrita. En este sentido, es sabido que factores atencionales y de recursos mnemónicos también afectan la producción textual, en lo referente a los aspectos ortográficos (Veys, 2009; Fayol \& Pacton, 2006).

Conscientes de que la escritura académica puede y debe ser enseñada en la Educación Superior (MesaValle \& Álvarez-Corral, 2010; Carlino, 2010) es que esta rúbrica de evaluación ha sido concebida a la vez como un útil de diagnóstico y como un dispositivo evaluativo. Esto la diferencia de otros instrumentos que ponen su énfasis sólo en este último aspecto. En este sentido, nos hacemos eco de la crítica a la evaluación a través de dispositivos tipo elección múltiple (Entwistle \& Karagiannopoulou, 2014; Scouller, 1998), optando por un método que si bien tiene sus dificultades (Torrubia \& Pérez, 2005) aporta una mirada más rica y compleja de este fenómeno. En efecto, la rúbrica cuenta con tres niveles de progresión en el dominio escritural: necesita reforzarse, parcialmente logrado y completamente logrado. Cada uno de estos niveles se encuentra debidamente explicitado, de manera que tanto el estudiante como el profesor cuenten con elementos que guíen la mejora en el proceso de apropiación de la escritura.

Esta grilla ha sido concebida para la escritura de un tipo preciso de texto: el ensayo académico. Definido como un tipo de texto más flexible y abierto, permite a quien lo escribe una aproximación más libre al objeto de discusión; es por esto que se trata de una de las formas más utilizadas por lo medios de comunicación en la cultura moderna. En términos retóricos, el ensayo se caracteriza por su forma inacabada, es decir, la redacción en proceso de una idea sin que en ésta se agote la misma (Sánchez, 2006). Es en ese sentido en el que, por vez primera, Montaigne utiliza la expresión para sus "Essaies" y es por estas razones que el 
ensayo se presenta como un buen recurso para el aprendizaje de la escritura académica, permitiendo la enseñanza de estrategias retóricas como la exposición y la argumentación. Como señaláramos de manera precedente, el ensayo permite pensar libremente sin agotar la idea en desarrollo. Desde esta perspectiva, quien escribe debe resolver un problema retórico (cómo comunicar de manera adecuada una idea, eficiente y eficazmente) y para ello debe dialogar consigo mismo, gracias al ejercicio escritural. La escritura deviene, en cierto modo, un ejercicio del pensamiento. El estudiante puede, así, aprender de manera más profunda, sobre la temática particular de la que habla, así como la manera más adecuada de comunicarla, logrando lo que Scardamalia y Bereiter (1987) llamaron "transformar el conocimiento".

\section{METODOLOGÍA}

Se describen a continuación la metodología del estudio, considerando el instrumento utilizado, la muestra del estudio, el protocolo de aplicación a los participantes, los criterios de evaluación y los procedimientos de validación de la propuesta.

El presente instrumento (tabla 1) fue concebido en el marco de un curso de expresión escrita para estudiantes de diversas carreras del área humanista de una Institución de Educación Superior chilena. Entre sus objetivos, destaca el de diagnosticar de manera precisa y comparativa el desempeño en escritura al inicio del ciclo universitario. Para controlar el efecto de la instrucción explícita en producción escrita y con el objeto de establecer una línea de base, la aplicación se realizó a comienzos del año lectivo. Tal y como señalado en la introducción, la estructura de la rúbrica reside en un modelo teórico que evalúa tanto los aspectos de organización y estructura del texto como asimismo, la forma redaccional para expresar las ideas y los aspectos formales de ortografía literal (puntual) y acentual, de manera concordante con las dimensiones expuestas en la introducción. Su concepción reside en el actual conocimiento de los procesos de escritura. Una versión preliminar de la rúbrica fue sometida a juicio de expertos.

La muestra del estudio, de carácter incidental, estuvo constituida por 745 estudiantes $(H=328, M=417)$ de primer año de universidad, provenientes de 17 carreras universitarias en el área de Humanidades y Ciencias sociales. El criterio de inclusión en la muestra fue ser universitario de primer año y estar inscrito en la asignatura de expresión escrita. Además, participaron como correctores 11 profesores del área de lengua castellana quienes recibieron dos sesiones de formación para capacitarlos en el uso de la rúbrica. En estas se verificó la calibración de los evaluadores en su uso. Sólo uno de los 11 evaluadores corresponde a uno autores de la rúbrica (Evaluador 7).

Tabla 1: Rúbrica de Evaluación Expresión Escrita - Ensayo

\begin{tabular}{|c|c|c|c|}
\hline Aspectos Evaluados & $\begin{array}{l}\text { Completamente Logrado } \\
3 \text { Puntos }\end{array}$ & $\begin{array}{l}\text { Parcialmente Logrado } \\
2 \text { Puntos }\end{array}$ & $\begin{array}{l}\text { Necesita Reforzarse } \\
1 \text { Punto }\end{array}$ \\
\hline $\begin{array}{l}\text { 1. Aspectos formales } \\
\text { Ortografía } \\
\text { ¿Hay errores de ortografía } \\
\text { literal? ¿Hay errores de } \\
\text { acentuación? ¿Hay errores } \\
\text { en la escritura de las } \\
\text { palabras? }\end{array}$ & $\begin{array}{l}\text { No existen errores } \\
\text { ortográficos literales, ni } \\
\text { acentuales, ni de escritura } \\
\text { de las palabras, El texto } \\
\text { está, en este sentido, } \\
\text { completamente limpio y } \\
\text { refleja una preocupación } \\
\text { por parte del autor y un } \\
\text { conocimiento de la norma } \\
\text { escrita, }\end{array}$ & $\begin{array}{l}\text { No hay errores de ortografía } \\
\text { literal, Existen algunos } \\
\text { errores de acentuación ( } 1 \text { a } \\
\text { 3) y en la escritura de las } \\
\text { palabras ( } 1 \text { a } 2 \text { ) pero que, } \\
\text { en ningún caso, dificultan la } \\
\text { lectura, ni generan } \\
\text { ambigüedad en la } \\
\text { interpretación del texto, }\end{array}$ & $\begin{array}{l}\text { Hay varios errores de } \\
\text { ortografía literal (más de 3), } \\
\text { de ortografía acentual (más } \\
\text { de } 3 \text { ) y en la escritura de las } \\
\text { palabras (más de } 2 \text { ). Incluso, } \\
\text { algunos errores dificultan la } \\
\text { lectura al funcionar como } \\
\text { elementos que la distraen y } \\
\text { al generar ambigüedades en } \\
\text { la interpretación del texto, }\end{array}$ \\
\hline 2. Contenidos del escrito & $\begin{array}{l}\text { En el escrito existe una } \\
\text { unidad de tema, }\end{array}$ & $\begin{array}{l}\text { En el escrito existe un tema } \\
\text { que se desarrolla, pero hay } \\
\text { digresiones en algunos de } \\
\text { los párrafos, }\end{array}$ & $\begin{array}{l}\text { No existe unidad de tema en } \\
\text { el escrito, Se tratan varios } \\
\text { tópicos al mismo tiempo, No } \\
\text { hay un eje temático central, }\end{array}$ \\
\hline $\begin{array}{l}\text { 2. } 1 \text { organización del texto } \\
\text { ¿Existe un tema claro y } \\
\text { preciso? ¿Es posible } \\
\text { reconocer las partes de } \\
\text { introducción, desarrollo y } \\
\text { conclusión? ¿Existe un } \\
\text { equilibrio adecuado entre } \\
\text { estas secciones? }\end{array}$ & $\begin{array}{l}\text { Existe un tema acotado, En } \\
\text { el texto es posible } \\
\text { reconocer párrafos que } \\
\text { cumplen las funciones de } \\
\text { introducción, desarrollo y } \\
\text { conclusión (apertura, } \\
\text { desarrollo y cierre } \\
\text { textuales), Existe un } \\
\text { adecuado equilibrio entre } \\
\text { cada una de estas } \\
\text { secciones, }\end{array}$ & $\begin{array}{l}\text { El tema no está bien } \\
\text { delimitado, En el texto es } \\
\text { posible reconocer párrafos } \\
\text { que cumplan las funciones } \\
\text { de introducción, desarrollo y } \\
\text { conclusión, pero no hay un } \\
\text { equilibrio adecuado entre } \\
\text { cada una de estas } \\
\text { secciones, porque algunas } \\
\text { están más desarrolladas } \\
\text { que otras, }\end{array}$ & $\begin{array}{l}\text { El tema es general, En el } \\
\text { texto es imposible reconocer } \\
\text { párrafos que cumplan las } \\
\text { funciones de introducción, } \\
\text { desarrollo y conclusión, ya } \\
\text { sea porque no cumplan las } \\
\text { tareas de apertura, } \\
\text { desarrollo o cierre o porque } \\
\text { si bien existe alguna sección } \\
\text { faltan otras, }\end{array}$ \\
\hline
\end{tabular}


Tabla 1 (continuación)

\section{1. 1. Introducción}

¿Están presentes en la introducción los siguientes elementos: antecedentes fundamentales sobre el tema, la tesis o afirmación principal, el objetivo del autor al escribir el texto? ¿La tesis se inserta de

\section{1. 2. Desarrollo}

¿Hay ideas fuerza que respalden la tesis 0 afirmación inicial del texto? ¿Se reconoce una ideas a lo largo del texto? ¿Están desarrolladas de manera equilibrada todas las ideas fuerza que fueron esbozadas a lo largo del texto o quedan ideas fuerza truncas?

\section{1. 3. Conclusión}

¿Hay en la conclusión una reformulación de la tesis? ¿Se verifica en la conclusión el paso de la tesis como afirmación inicial a una tesis confirmada por el desarrollo del texto? ¿La estrategia de cierre incluye: síntesis de lo afirmado anteriormente $y$ replanteamiento del problema de manera original?

\section{2. Conexión entre párrafos}

\section{¿Se conectan}

temáticamente los párrafos entre sí de manera coherente? ¿Se introduce la idea fuerza al inicio de cada párrafo correspondiente? manera clara en el tema? progresión coherente de las

En la introducción se verifica la presencia de antecedentes fundamentales sobre el tema, la tesis y el objetivo del escritor del texto, La tesis se vincula claramente con el tema,

Existen ideas fuerza que respaldan la tesis inicial del texto, Se reconoce una progresión coherente de las ideas a lo largo del texto, Todas las ideas fuerza que fueron esbozadas están desarrolladas de manera equilibrada, ninguna de las ideas fuerza queda trunca,

En la conclusión hay reformulación de la tesis, Se verifica en la conclusión el paso de la tesis inicial a una tesis confirmada, La estrategia de cierre incluye: síntesis de lo afirmado anteriormente $y$ replanteamiento del problema de manera original,

Los párrafos se conectan temáticamente entre sí de manera coherente, Las ideas fuerza de cada párrafo tienen relación con el resto de las ideas de los otros párrafos y con la tesis o afirmación global del texto, La idea fuerza de cada párrafo se introduce al inicio lo que favorece la comprensión del texto,

\subsection{Conexión entre} oraciones

¿Cada una de las oraciones se vincula con la que antecede? ¿La puntuación favorece la conexión entre las oraciones? ¿En general, el texto incurre en errores de puntuación que dificultan la lectura?
Cada una de las oraciones se vincula con la que le antecede, La puntuación favorece la conexión entre las oraciones, En general, el texto no presenta errores de puntuación que dificulten la lectura,
En la introducción se verifica la presencia de la tesis pero no hay antecedentes fundamentales sobre el tema ni se señala el objetivo del escritor del texto, La tesis se vincula con el tema de manera indirecta,

Hay ideas fuerza que

respaldan la tesis inicial del texto, Pero no se evidencia una progresión coherente de las ideas a lo largo del texto, No todas las ideas fuerza que fueron esbozadas a lo largo del texto son desarrolladas de manera equilibrada e, incluso, algunas ideas fuerza quedan truncas (1 a 2),

En la conclusión hay una reformulación de la tesis, Se verifica en la conclusión el paso de la tesis inicial a una tesis confirmada, La estrategia de cierre incluye una síntesis de lo afirmado anteriormente pero no hay un replanteamiento del problema de manera original, solo se vuelve a repetir el planteamiento inicial,

Los párrafos se conectan temáticamente entre sí de manera coherente pero la idea fuerza de cada párrafo no se introduce al inicio, Esto dificulta en parte la comprensión del texto al requerir un esfuerzo adicional del lector,

En la introducción no se verifica la presencia de antecedentes fundamentales sobre el tema ni el objetivo del escritor del texto, La tesis no se vincula con el tema o simplemente no existe,

No hay ideas fuerza que respalden la tesis inicial del texto, El texto no presenta ideas que justifiquen la postura del escritor, Este último, solo se limita a repetir de manera redundante su posición respecto del tema, pero sin justificarla o respaldarla,

En la conclusión no hay una reformulación de la tesis, No se verifica en la conclusión el paso de la tesis como afirmación inicial a una tesis, La estrategia de cierre no incluye ni síntesis ni replanteamiento del problema, La carencia de cierre redunda en la dificultad para comprender el contenido global del escrito,

Los párrafos no se conectan temáticamente entre sí de manera coherente, No existen elementos que permitan al lector asignarle una unidad al texto, Este último se percibe como un conglomerado de información dispersa,
El texto presenta pocos errores de conexión entre las oraciones (1 a 2), Los errores se deben al uso incorrecto de cláusulas subordinadas o a errores de puntuación: omisión de punto aparte o separación con coma de sujeto $y$ predicado, En general, la puntuación favorece la conexión entre oraciones, solo hay pocos errores que dificulten la lectura (1 a 2),
El texto presenta errores de conexión entre las oraciones (más de 2), Los errores no solo se deben al uso incorrecto de cláusulas subordinadas o a errores de puntuación (omisión de punto aparte o separación con coma de sujeto y predicado), sino que al empleo incorrecto o la falta de un conector, La puntuación no favorece la conexión entre oraciones, hay errores que dificultan la lectura (más de 2), 
Tabla 1 (continuación)

\begin{tabular}{|c|c|c|c|}
\hline $\begin{array}{l}\text { 2.4. Estilo } \\
\text { ¿El texto tiene un } \\
\text { vocabulario variado en el } \\
\text { uso de sustantivos, } \\
\text { adjetivos y verbos? ¿Existe } \\
\text { variedad de conectores? } \\
\text { ¿Se escribe de manera } \\
\text { informal, con giros } \\
\text { coloquiales? ¿Hay variedad } \\
\text { de puntuación? }\end{array}$ & $\begin{array}{l}\text { El texto tiene un vocabulario } \\
\text { variado en el uso de } \\
\text { sustantivos, adjetivos y } \\
\text { verbos, Para un mismo } \\
\text { concepto se emplean } \\
\text { diferentes palabras evitando } \\
\text { la repetición innecesaria de } \\
\text { vocablos, Existe variedad } \\
\text { de conectores, Para una } \\
\text { misma función lógica se } \\
\text { emplean diferentes } \\
\text { elementos ilativos evitando } \\
\text { su reiteración innecesaria, } \\
\text { Se escribe de manera } \\
\text { formal sin giros coloquiales, } \\
\text { Hay un uso variado de } \\
\text { puntuación no solo se } \\
\text { emplea coma y punto, sino } \\
\text { que también dos puntos y } \\
\text { punto y coma, }\end{array}$ & $\begin{array}{l}\text { El texto tiene un vocabulario } \\
\text { variado en el uso de } \\
\text { sustantivos, adjetivos y } \\
\text { verbos, pero no existe } \\
\text { variedad de conectores, } \\
\text { reiterando siempre los } \\
\text { mismos, Se escribe de } \\
\text { manera formal sin giros } \\
\text { coloquiales, pero hay un uso } \\
\text { poco variado de puntuación, } \\
\text { solo se usa coma y punto, }\end{array}$ & $\begin{array}{l}\text { El texto no tiene un } \\
\text { vocabulario variado en el } \\
\text { uso de sustantivos, adjetivos } \\
\text { y verbos, No existe } \\
\text { variedad de conectores, Se } \\
\text { escribe de manera informal } \\
\text { con giros coloquiales, No } \\
\text { hay un uso variado de } \\
\text { puntuación, En este sentido, } \\
\text { el autor no marca la } \\
\text { diferencia entre la } \\
\text { conversación oral y las } \\
\text { normas del código escrito, }\end{array}$ \\
\hline
\end{tabular}

El procedimiento para evaluar la escritura, consistió en invitar a los estudiantes -durante la sesión inicial del curso- a la lectura del extracto de un texto que tenía por objetivo situarlos de manera amplia en una temática específica. Sabiendo que los conocimientos previos, así como la memoria pueden jugar un rol capital en la escritura (Hayes, 2010), se concibió el pre-texto de escritura como un mecanismo que asegurar una línea de base común entre los estudiantes respecto de sus conocimientos en el tema. Posterior a la lectura de éste, se les invitó a redactar un escrito de 2 o 3 carillas en donde se expresara la propia opinión acerca de una afirmación sacada del pre-texto. Dicho enunciado planteaba dos puntos de vista sobre un tema (vigencia de la música chilena) y conminaba a tomar partido, al menos, por alguna de las dos posturas. La consigna de carácter abierto (ver Figura 1), sólo limitaba el tiempo disponible, la longitud del escrito y el contenido mínimo.

Producción de un texto (60 minutos)

"Escribe un texto, de entre 2 y 3 páginas, en el que desarrolles tu opinión acerca del tema que se presenta a continuación."

Algunos autores, como Larraín, afirman que la música tradicional chilena ha sido desplazada por ritmos de origen extranjero. Otros sostienen que no es más que una moda pasajera, prueba de ello son los grupos que han revitalizado nuestro baile nacional a través de las llamadas "cuecas choras". ¿Crees que la música chilena se ha visto relegada a un segundo plano? Fundamenta tu respuesta."

Fig. 1: Consigna de producción de un texto

Como criterios de evaluación, la rúbrica de corrección (ver Tabla 1) distingue para cada aspecto tres niveles de desempeño (el mejor recibía 3 puntos y el más deficiente, 1). Estos niveles son: completamente logrado (3 puntos), es decir, cuando el texto evaluado cumple con los estándares mínimos en el cumplimiento del rasgo que se evalúa. Parcialmente logrado (2 puntos) caracteriza un punto intermedio en el cual se observan ciertas carencias o falta importantes en relación al aspecto evaluado (definidas previamente en la rúbrica), pero al mismo tiempo cumple con otras. Finalmente, se evaluará el texto como necesita reforzarse (1 punto), cuando el escrito no cumpla con los estándares fundamentales del rasgo evaluado. De esta forma, el puntaje máximo posible es de 27 puntos.

Para efectos de administración de la prueba, se contó con la aplicación de la misma en el marco de un curso mínimo de primer año. La prueba fue administrada de manera colectiva -en cada clase- bajo la supervisión de 1 evaluador y 2 ayudantes. El tiempo destinado realizar la prueba fue de 70 minutos que consideraba $10 \mathrm{~min}$. para la lectura previa del pre-texto y 60 minutos para la elaboración del texto.Tras la aplicación, se procedió a evaluar de manera exploratoria las características psicométricas de la rúbrica, específicamente: la validez de constructo, a través del análisis y la contrastación de la estructura factorial que arroja el análisis factorial de componentes principales (AF), con respecto a los fundamentos teóricos que están a la base del diseño de la rúbrica. En segundo término, se analizó la confiabilidad del test a través del alfa de Cronbach (Barbaranelli, Lee, Vellone, \& Riegel, 2015; Cortina, 1993; Streiner, 2003) para la 
prueba en su conjunto y para cada uno de los evaluadores (correctores) del test, contrastándose sus respectivos alfas a través de un utilitario computacional para comparar alfas de Cronbach en muestras independientes (Valenzuela, 2007) basado en el trabajo de Hakstian \& Whalen (1976).

Finalmente, realizamos un análisis respecto del nivel de discriminación de la rúbrica. Como puede observarse en la Tabla 4 , todos los índices D son iguales o superiores a 0.43 y pueden ser considerados como excelentes bajo los criterios de Ebel y Frisbie (1986; Khan, Ishrat, \& Khan, 2015). Lo anterior muestra, por tanto, que la prueba posee un muy buen nivel de discriminación.

\section{RESULTADOS}

El análisis de la estructura factorial de la rúbrica se realizó a través de un Análisis factorial de componentes principales $(\mathrm{AF})$, utilizando una rotación Promax (kappa $=4$ ), dada la intercorrelación significativa entre los ítemes. En la tabla 2, es posible observar el detalle. Por razones de legibilidad, se omiten las puntuaciones factoriales menores a 0,3. Por otra parte, el análisis de los datos del test de esfericidad de Bartlett $\left(x^{2}=\right.$ $4148,880 \mathrm{p}<.001)$ y el índice de Kaiser-Meyer-Olkin $(\mathrm{KMO}=.921)$ muestran que los datos se adecuan de muy buena forma al análisis factorial. Adicionalmente, se constata que las comunalidades iniciales, es decir, la fracción de variabilidad de cada elemento que se ha explicado con el modelo de factores, son bastante altas, siendo todos autovalores superiores a 0.627 .

Tabla 2: Análisis factorial, Matriz Rotada (Promax), cargas factoriales,.

\begin{tabular}{|l|l|l|}
\hline \multirow{2}{*}{ Aspectos evaluados } & Componentes \\
\cline { 2 - 3 } & 1 & 2 \\
\hline Unidad de Contenido & .714 & \\
\hline Organización del ensayo & .972 & \\
\hline Introducción & .928 & \\
\hline Desarrollo del tema & .824 & \\
\hline Conclusión & .885 & \\
\hline Conexión entre párrafos & .601 & .314 \\
\hline Conexión entre frases & & .749 \\
\hline Riqueza de estilo & & .689 \\
\hline Ortografía & & .914 \\
\hline
\end{tabular}

La solución rotada del Análisis factorial (ver Tabla 2 y Figura 2) muestra dos factores principales que, en su conjunto explican un $70.9 \%$ de la varianza y que tienen una correlación entre ellos de $r=.583$. El primero de los factores, que explica un $58.897 \%$ de la varianza, está constituido por el conjunto de aspectos que evalúan los rasgos globales de la estructura y organización del texto, es decir: Unidad de contenido (cont), organización del ensayo (org), introducción (intro), desarrollo del tema (des), conclusión (conc) y conexión entre los párrafos (conexp). Por su parte, el segundo factor, que aporta un $12.029 \%$ a la explicación de la varianza, agrupa aquellos aspectos que contribuyen a la coherencia local, a saber la conexión entre las frases (conexph), estilo y ortografía (af).

El análisis de confiabilidad de la rúbrica muestra, para el primer factor (los rasgos globales de la estructura y organización del texto ) un alfa de Cronbach de $\alpha=.923$ mientras que en el segundo factor (de coherencia local) este indicador es $\alpha=.717$. Ahora bien, dado el posible sesgo entre los distintos evaluadores en la utilización de la misma, hemos procedido a analizar la confiabilidad para cada uno de ellos. Los resultados de este análisis muestran que todos los índices alfa son superiores a .73, estando la mayor parte en torno o sobre a .8 (ver Tabla 3). Estos resultados son normalmente considerados como buenos en Ciencias Sociales (Cortina, 1993; Streiner, 2003). Así, el nivel de homogeneidad obtenido en este índice apoya uno de los aspectos fuertes de esta rúbrica, cual es el de permitir una medición con altos niveles de precisión, independientemente del evaluador.

No obstante, si bien todos los índices alfa son bastante altos, encontramos diferencias significativas entre ellos (X2 = 29.47093; $8 \mathrm{gl} ; \mathrm{p}=.001$ ). De los 55 pares de comparaciones posibles 20 tienen diferencias estadísticamente significativas. De éstas, 9 son significativas con un $p \leq .01$, mientras que las restantes lo son con un $p \leq .05$, no hallándose significaciones menores a $p \leq .001$. Las diferencias se relacionan fundamentalmente con los valores extremos, matizadas por el efecto del tamaño de la muestra asociado a cada uno de los evaluadores. 
Tabla 3: Síntesis de diferencias significativas de alfas entre evaluadores

\begin{tabular}{|l|l|l|l|l|l|l|}
\hline \multirow{2}{*}{ Evaluador } & \multicolumn{2}{|l|}{ Alpha de Cronbach } & \multirow{2}{*}{ X2 } & \multirow{2}{*}{ Sig. } \\
\cline { 2 - 5 } & $\mathrm{\alpha} 1$ & $\mathrm{n}$ & $\mathrm{\alpha} 2$ & $\mathrm{n}$ & & \\
\hline Evaluador 1 - Evaluador 3 & .732 & 34 & .887 & 87 & 6.71207 & .010 \\
\hline Evaluador 1 - Evaluador 5 & .732 & 34 & .897 & 73 & 7.82085 & .005 \\
\hline Evaluador 1 - Evaluador 6 & .732 & 34 & .869 & 88 & 4.75737 & .029 \\
\hline Evaluador 1 - Evaluador 7 & .732 & 34 & .904 & 59 & 8.50951 & .004 \\
\hline Evaluador 1 - Evaluador 8 & .732 & 34 & .871 & 68 & 4.68505 & .030 \\
\hline Evaluador 2 - Evaluador 3 & .779 & 71 & .887 & 87 & 7.34871 & .007 \\
\hline Evaluador 2 - Evaluador 5 & .779 & 71 & .897 & 55 & 7.88730 & .005 \\
\hline Evaluador 2 - Evaluador 6 & .779 & 71 & .869 & 88 & 4.54368 & .033 \\
\hline Evaluador 2 - Evaluador 7 & .779 & 71 & .904 & 59 & 9.67573 & .002 \\
\hline Evaluador 2 - Evaluador 8 & .779 & 71 & .871 & 68 & 4.33997 & .037 \\
\hline Evaluador 3 - Evaluador 10 & .887 & 87 & .799 & 112 & 7.17587 & .007 \\
\hline Evaluador 3 - Evaluador 11 & .887 & 87 & .796 & 52 & 4.61935 & .032 \\
\hline Evaluador 3 - Evaluador 4 & .887 & 87 & .816 & 73 & 4.01417 & .045 \\
\hline Evaluador 4- Evaluador 5 & .816 & 73 & .897 & 55 & 4.64007 & .031 \\
\hline Evaluador 4- Evaluador 7 & .816 & 73 & .904 & 59 & 6.02480 & .014 \\
\hline Evaluador 5 - Evaluador 10 & .897 & 55 & .799 & 112 & 7.60096 & .006 \\
\hline Evaluador 5 - Evaluador 11 & .897 & 55 & .796 & 52 & 5.24991 & .022 \\
\hline Evaluador 6 - Evaluador 10 & .869 & 88 & .799 & 112 & 3.99007 & .046 \\
\hline Evaluador 7 - Evaluador 10 & .904 & 59 & .799 & 112 & 9.69768 & .002 \\
\hline Evaluador 7 - Evaluador 11 & .904 & 59 & .796 & 52 & 6.51841 & .011 \\
\hline
\end{tabular}

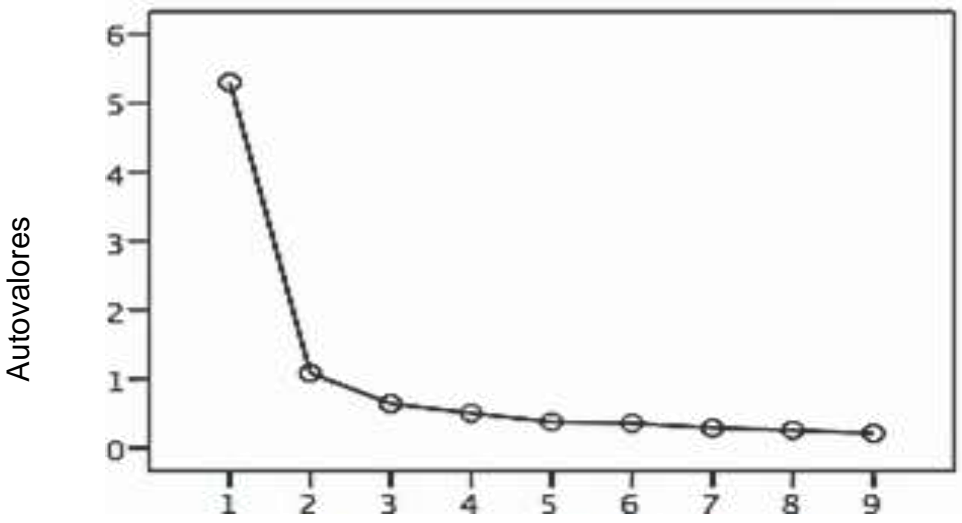

Número de componentes

Fig. 2: Gráfico de sedimentación, A. Factorial

Como consideración adicional, cabe destacar que el evaluador 7 corresponde a uno de los creadores de este instrumento y que si bien tiene diferencias significativas con 5 de otros 10 evaluadores, no la tiene con la otra mitad de los evaluadores que corresponden a académicos que fueron capacitados en la utilización de la rúbrica, pero que no han tenido una vinculación directa en la elaboración del instrumento, como en el caso del evaluador 7 . Lo anterior, nos sugiere que la capacitación en el uso de la rúbrica puede lograr estándares más que suficientes para la utilización adecuada de este instrumento.

En relación al nivel de dificultad de los aspectos evaluados por la rúbrica, el que aparece como mejor logrado es el de unidad de contenido, mientras que los que implican una mayor dificultad para esta muestra de alumnos son, en primer lugar, la ortografía seguida de la capacidad de redactar adecuadamente la conclusión del texto. 
Tabla 4: Media, desviación estándar e índice de discriminación por aspecto evaluado

\begin{tabular}{|l|l|l|l|}
\hline Ítem & media & S.D & Índice de Disc. \\
\hline Unidad de contenido & 2.0913 & .77059 & 0.84 \\
\hline Organización del ensayo & 1.8295 & .71781 & 0.83 \\
\hline conexión entre párrafos & 1.7302 & .74665 & 0.81 \\
\hline Desarrollo del tema & 1.8081 & .76863 & 0.77 \\
\hline Introducción & 1.8268 & .75194 & 0.72 \\
\hline Conclusión & 1.7812 & .77193 & 0.69 \\
\hline Conexión entre frases & 1.7289 & .68904 & 0.69 \\
\hline Riqueza de estilo & 1.6067 & .74394 & 0.56 \\
\hline Ortografía & 1.5128 & .71667 & 0.43 \\
\hline Promedio & 1.7684 & 0.7419 & 0.71 \\
\hline
\end{tabular}

Finalmente, realizamos un análisis respecto del nivel de discriminación de la rúbrica. Como puede observarse en la Tabla 4, todos los índices $\mathrm{D}$ son iguales o superiores a 0.43 y pueden ser considerados como excelentes bajo los criterios de Ebel y Frisbie (1986; Khan, Ishrat, \& Khan, 2015). Lo anterior muestra, por tanto, que la prueba posee un muy buen nivel de discriminación.

\section{DISCUSIÓN}

El objetivo de este artículo ha sido explorar las características psicométricas de una rúbrica de expresión escrita de nivel académico. El estudio psicométrico de la rúbrica fue realizado en una población de 745 estudiantes universitarios de primer año, los cuales fueron sometidos al ejercicio de escribir un ensayo académico. A través de este diagnóstico, se esperaba evaluar el nivel inicial de habilidades de escritura académica de los estudiantes que ingresan al ciclo superior de educación, para remediar -a través de la intervención en un curso especialmente diseñado- los déficits evidenciados por este proceso diagnóstico. El análisis de las características psicométricas de la rúbrica permite afirmar que este instrumento es capaz de evaluar de manera valida y confiable la competencia de expresión escrita en alumnos que ingresan al ciclo superior de educación.

En primer término, el análisis de confiabilidad muestra que la rúbrica es un instrumento que mide con precisión ambas dimensiones de la escritura académica de tipo ensayo. Del mismo modo, al ser puesta a prueba la consistencia entre los correctores, se comprueba que la rúbrica es altamente fiable, incluso, al ser utilizada por dos o más correctores. En segundo término, el análisis de la estructura factorial de la rúbrica confirma que el constructo "escritura académica" puede ser concebido como una estructura doble, distinguiéndose aspectos locales y globales del texto. En efecto, escribir es un proceso altamente complejo que conjuga habilidades de diverso orden. En el análisis factorial exploratorio de la rúbrica, efectivamente observamos, por un lado, la agrupación de los aspectos locales del proceso de escritura (ortografía acentual y puntual, vocabulario, coherencia entre las frases), mientras que en otro componente encontramos elementos de orden global (unidad temática, organización del texto, introducción, desarrollo del tema, conclusión y coherencia entre párrafos). La conjugación de ambos aspectos da como resultado una estructura coherente y cohesiva, esto es, un texto.

Los análisis asociados a la estructura factorial de la rúbrica nos permiten, asimismo, inferir que en todo proceso escritural el escritor se comporta también como lector de su propio texto en el proceso de revisión y, que en niveles iniciales -como es el caso de estudiantes que ingresan al Ciclo superior de educación-, cierto aspectos devienen más dificultosos que otros.

En este sentido, no es raro que la ortografía (puntual y acentual) sean menos logradas por los estudiantes de la muestra. Es sabido que en tareas de alta demanda cognitiva (como lo es la escritura de un texto) los escritores pueden destinar una gran carga atencional a los aspectos de coherencia global, descuidando aspectos locales como la ortografía (Chanquoy \& Alamargot, 2003). Similares son los resultados obtenidos en tareas experimentales con el paradigma de la doble tarea (Chanquoy \& Alamargot, 2003; Hupet, Schelstraete, Demaeght, \& Fayol, 1996; Hupet \& Veys, 2012; Piolat \& Olive, 2000). Estos datos nos obligan a considerar las características de la escritura en un contexto de formación, en donde la tarea a la vez de representar un útil de comunicación, constituye una herramienta del pensamiento, por medio de la cual el estudiante da cuenta y cae en la cuenta de lo que sabe y de cómo lo comunica de manera eficaz. 
Finalmente, creemos que las bondades de utilizar este instrumento para medir expresión escrita no se remiten sólo a la evaluación diagnóstica de la escritura como habilidad específica, a la enseñanza del pensamiento a través de la escritura, sino también al dominio progresivo de la misma en un contexto académico.

\section{CONCLUSIONES}

El presente estudio ofrece un instrumento para evaluar la producción escrita en el ámbito académico. Las características psicométricas de esta rúbrica para evaluar escritura académica a nivel universitario hacen de este instrumento, no sólo una herramienta válida, confiable y con altos niveles de discriminación, sino que además posibilita, previo entrenamiento de los evaluadores, una alta consistencia en los índices de confiabilidad intercorrectores.

Incluir la escritura en todas las materias de formación, requiere de instrumentos que posibiliten el diagnóstico y seguimiento de nuestros estudiantes en sus aprendizajes. La escritura como herramienta epistémica permite al que escribe reflexionar y tomar distancia del objeto descrito y del mismo modo, resituarlo y comprender en mayor profundidad articulando sus conocimientos con nuevas fuentes. Como señala Carlino (2005), al escribir nuestros estudiantes no solo están siendo evaluados en sus conocimientos, la forma en que están expresadas las ideas, la articulación de las ideas, la argumentación son elementos esenciales que dan cuenta de la profundidad de tal conocimiento.

\section{REFERENCIAS}

Adoumieh, N., Escribir desde las disciplinas: Una experiencia pedagógica en ciencias sociales, Paradigma, 35(1), 183-213, (2014)

Alamargot, D. y L. Chanquoy, Through the models of writing, 4-271. Kluwer Academic Publishers, Dordrecht, Holanda, (2001)

Barbaranelli, C., y otros tres autores, The Problem With Cronbach's Alpha: Comment on Sijtsma and van der Ark,. Nursing Research, 64(2), 140-145, (2015)

Bazerman, C., Reference guide to writing across the curriculum, 5-175. Parlor Press, Anderson SC, USA, (2005)

Bereiter, C. y M. Scardamalia, The psychology of written composition, 4-390, Laurence Erlbaum Associates, Mahwah, New Jersey, USA, (1987)

Cabrera, M., El instrumento CODICE-Derecho y su aplicación en la Facultad de Derecho de la Universidad de Chile, Revista de Pedagogía Universitaria y Didáctica del Derecho, 1(1), 2-19, (2014)

Carlino, P. Escribir, leer y aprender en la universidad, 9-200, Una introducción a la alfabetización académica. Buenos Aires, Fondo de Cultura Económica, (2005)

Carlino, P., "Reading and writing in the social sciences in Argentine universities". In Traditions of writing research, by C. Bazerman, R. Krut, K. Lunsford, S. McLeod, S. Null, P. Rogers, \& A. Stansell (Eds.), pp. 166-180). London, UK., Routledge, (2010). (pp. 283-296). London: Routledge, (2010)

Carlino, P., Alfabetización académica diez años después, Revista Mexicana de Investigación Educativa, 18(57), 355-381, (2013)

Carlino, P., El proceso de escritura académica: cuatro dificultades de la enseñanza universitaria, Educere, 8(26), 321-327, (2004)

Carlino, P., Representaciones sobre la escritura y formas de enseñarla en universidades de América del Norte, Revista de Educación (336), 143-168, (2005)

Chanquoy, L. y D. Alamargot, Mise en place et développement des traitements rédactionnels: le rôle de la mémoire de travail, Le Langage et l'homme, 37(2), 171-190, (2003)

Cortina, J., What is coefficient alpha? An examination of theory and applications, Journal of Applied psychology, 78(1), 98-104, (1993)

Ebel, R., y Frisbie, D, Essential of Educational Measurement, 1-370, Prentice- Hall, Englewood Cliff, NJ, USA, (1986)

Entwistle, N. y E. Karagiannopoulou, "Perceptions of Assessment and their Influences on Learning". In C. Kreber, C. Anderson, N. Entwhistle, y J. McArthur (Eds.), Advances and Innovations in University Assessment and Feedback, (pp. 75-98), Edinburgh University Press, Edimburgo, Inglaterra, (2014) 
Fayol, M., y Pacton, S. L'accord du participe passé: entre compétition de procédures et récupération en mémoire. Langue française, 151(3), 59-73, (2006)

Findley, W. G. A rationale for evaluation of item discrimination statistics, Educational and Psychological Measurement 16,175-180, (1956)

Flotts, P., y J. Manzi, Medición de habilidades de comunicación escrita en estudiantes universitarios, La experiencia UC. http://goo.gl/1iPfji . Acceso: 4 abril, (2012)

Garcés, C. R. y V. C. Riquelme, Calidad en la formación inicial docente: los déficits de las competencias pedagógicas y disciplinares en Chile, Actualidades Investigativas en Educación, 14(2), 1-25, (2014)

Hakstian, R., y T. Whalen, A K-sample significance test for independent Alpha coefficients, Psychometrika, 41, 219-231, (1976)

Hayes, J. R., y Berninger, V. W. "Relationships between idea generation and transcription". In Traditions of writing research by C. Bazerman, R. Krut, K. Lunsford, S. McLeod, S. Null, P. Rogers, \& A. Stansell (Eds.), pp. 166-180). London, UK., Routledge, (2010)

Hupet, M. y E. Veys, Approche cognitive de la gestion et du contrôle de l'orthographe grammaticale, Approche neuropsychologique des apprentissages chez l'enfant 116, 54-59, (2012)

Hupet, M., y otros tres autores, Les erreurs d'accord sujet-verbe en production écrite, L'année psychologique, 96(4), 587-610, (1996)

Kelley, T. L., Selection of upper and lower groups for the validation of test items, Journal of Educational Psychology, 30, 17-24, (1939)

Khan, G.-A. N., Ishrat, N., y Khan, A. Q., Using item analysis on essay types questions given in summative examination of medical college students: facility value, discrimination index, doi: 10.5455/23206012.ijrms20150131, Int J Res Med Sci., 3(1), 178-182, (2015)

Lieuw, W. M., y Ball, A. F. "Social academic writing. Exploring academic literacies in text-based computer conferencing" In Traditions of writing research by C. Bazerman, R. Krut, K. Lunsford, S. McLeod, S. Null, P. Rogers, \& A. Stansell (Eds.), pp. 166-180). London, UK., Routledge, (2010). (pp. 324-338), Routledge, London, Inglaterra, (2010)

Melas, M y otros tres autores, Percepción de Autoeficacia en la Solución de Problemas y Comunicación Científica en Universitarios de Ingeniería y Ciencias Sociales, doi:10.4067/S0718-50062015000400011, Formación Universitaria 8(4)93-100, (2015)

Mesa-Valle, M y Álvarez-Corral, M. Nuevos Recursos Docentes Aplicados en Asignaturas de la Titulación de Ciencias Ambientales, Formación Universitaria, 3(3),15-22, (2010)

Piolat, A., y T. Olive, Comment étudier le coût et le déroulement de la rédaction de textes? La méthode de la triple tâche: Un bilan méthodologique, L'année psychologique, 100(3), 465-502, (2000)

Rodríguez, C. y V. Castillo, Calidad en la formación inicial docente: los déficits de las competencias pedagógicas y disciplinares en Chile, Actualidades Investigativas en Educación, 14(2) 1-25, (2014)

Sánchez, J., (Ed.). Saber escribir, 1-513. Aguilar, Buenos Aires, Argentina, (2006)

Scouller, K., The influence of assessment method on students' learning approaches: Multiple choice question examination versus assignment essay, Higher Education, 35(4), 453-472, (1998)

Streiner, D. L., Starting at the Beginning: An Introduction to Coefficient Alpha and Internal Consistency. Journal of Personality Assessment, 80(1), 99-103, (2003)

Torrance, M. y D. Galbraith, "The processing demands of writing" In Handbook of writing research by C. MacArthur, S. Graham, y J. Fitzgerald (Eds.), pp. 67-80, Guilford, New York, USA, (2006)

Torrubia, R. y J. Pérez, La difícil objetividad de las pruebas de ensayo en la evaluación del rendimiento académico, Educación médica, 8(1), 17-21, (2005)

Valenzuela, J., Alpha comparator [computer software]. Université Catholique de Louvain, Louvain la Neuve, Bélgica, (2007)

van Eemeren, F. H. (Ed.). Crucial concepts in argumentation theory, (1-236), Amsterdam University Press, Amsterdam, Holanda, (2001) 